intelligence. Francis Galton in the last century argued that high population intelligence was an important requirement for the achievement of a civilization with high economic, cultural and intellectual attainments. This theme was re-stated in the 1930s by Cyril Burt and E.L. Thorndike and more recently by R.B. Cattell and John Baker. It was belief in the social value of intelligence that gave rise to concern that dysgenic trends might be operating in advanced societies, with the consequent decline of national levels of intelligence. It might have been expected that a handbook aiming at comprehensive coverage would have provided some discussion of these important questions.

A second omission concerns the physiology of intelligence. The discovery during the last decade of the high correlation between IQ and the EEG evoked potential promises to be one of the significant developments in our understanding of the brain mechanisms underlying intelligence; it is a pity that there is no mention of this work.

Finally, and not trivially, the index is totally inadequate. An index normally runs to about two pages per one hundred pages of text. In this volume the index consists of barely four pages to service a thousand pages of text. The result is that there is no appearance in the index of many important topics discussed in the text, even including $g$ (general intelligence), arguably the most important concept ever formulated in this field. It is a matter for astonishment that the publishers and editor - no doubt people of considerable ability - are apparently not aware that a handbook must have a good index if it is to serve its purpose. Such are the enigmas of intelligence.

Richard Lynn is Professor of Psychology at the New University of Ulster, Coleraine.

\section{Sea changes}

\section{A.J. Southward}

\section{Climate and Fisheries.}

By D.H. Cushing.

Academic: 1983. Pp.372. £28, \$49.50.

History shows how the fortunes of towns and states rose and fell with the success or failure of the great northern fisheries, such as cod and herring. Many reasons have been advanced for the variations in abundance and occurrence of the shoals, but changes in weather and climate have long been the favourite, and fishery scientists have to spend much time assessing fluctuations in sea temperature, water currents and winds. Dr Cushing is particularly fitted to write on this subject; he is well known for his studies of the impact of recent climatic changes on marine life, and is also an expert on production processes in the sea, especially the links between algal growth, herbivore grazing and young fish survival, one of the mechanisms controlling the abundance of the shoals.

The first four chapters introduce the subject: a brief summary of the major fisheries and their technologies; a survey of the way in which different fishes spawn, grow and migrate; a discussion of past changes; and a review of the meteorological background to climatic change. The author then moves deeper into his subject to describe the advance north of invertebrates and fish during the warm period between 1920 and 1960. This is followed with a review of all aspects of short-term fluctuation in fish stocks, which the author would evidently like to relate to year-to-year differences in phytoplankton production. There are other chapters dealing in detail with changes in the North Sea and the Baltic, perhaps the best studied seas in the world, and a description of the effects of very cold winters.

Dr Cushing devotes a whole chapter to the sorry tale of the Peruvian anchoveta fishery, which collapsed in 1972 after heavy fishing during a low point in a natural fluctuation. $\mathrm{He}$ notes how researches since then have increased our knowledge of hydrographic events in the Pacific to the point where it is now possible to predict intensification of the south-going warm current (the so-called El Niño) that drives the fish away and reduces recruitment. However he does not rub home sufficiently the message that continued overfishing contributed to the collapse, as happened with other fish that vanished during a period of natural decline, such as the California sardine.

Of course, the author's principal concern, as he stresses, is with natural changes. In the final chapters he discusses the mechanisms of natural change and the consequences from the point of view of fishery management. Many of the world's fisheries are now in a state of decline due to over-exploitation. It is vitally important that fishery managers should be able to distinguish between natural fluctuations and man-made changes, and assess the risks of allowing continued fishing on a smaller and fluctuating stock.

There are a number of irritating minor blemishes, including persistent misspellings, misunderstandings of other authors' work and garbled paragraphs, suggesting failure to consult primary sources and lack of critical reading of the manuscript. In addition, the author's terse style makes little concession to the nonspecialist reader, and beginners must look elsewhere for enlightenment. These, however, are relatively minor drawbacks. The book provides a fact-filled summary that is essential for all marine scientists, and is a must for those concerned with fishery management, environmental assessment and political economics.

A.J. Southward is at the Marine Biological Association's Laboratory, Plymouth.
Neuroscience meets immunology

\section{Ron McKay}

Neuroimmunology.

Edited by Jeremy Brockes.

Plenum: 1982. Pp.256. \$29.50, £20.65.

THERE have been two major technical developments in molecular biology in recent years - recombinant DNA and hybridoma technology. Both of these techniques are only just beginning to be applied to the outstanding problems in neurobiology, but already they promise to revolutionize our understanding in many areas of neuroscience.

In this book, Jeremy Brockes has gathered eight contributions which discuss the use of immunological techniques to study the nervous system. The primary reason for using such procedures is the exquisite specificity with which antibody can bind to antigen. However, until recently the complex array of antibodies produced in an immune response was the main difficulty in applying immunoglobulins (which bind specifically to antigens) to probe biological structures. The solution to the problem was the experiment of Kohler and Milstein, which showed that lymphocytes secreting different antibodies could be rescued from a rapid death in culture if they were fused with myeloma cells and could then be separated to provide access to the component antibodies. This experiment, which grew out of a marriage between immunology and somatic cell genetics, gave us fresh access to the large number of specific antibodies synthesized by the vertebrate immune system.

There are many applications of immunological technology to studies of the nervous system. In this book two chapters are devoted to the use of antibodies in identifying and characterizing the components of the neuromuscular junction. A third chapter discusses the application of monoclonal antibodies to map synaptic constituents of the vertebrate central nervous system. Three further contributions consider the use of monoclonal antibodies in defining the cellular heterogeneity among neurons and glia, these chapters being buttressed by an account of the strikingly successful use of specific immunoglobulins to identify, purify and functionally define subsets of cells in the immune system. Finally, the pathologic interaction of the immune system and the nervous system is considered. As indicated by the variety of the contributions in the book, the term neuroimmunology itself is vague in meaning; but common to all of the chapters is the sense of excitement over the early successes of this means of identifying specific molecules in a complex tissue which is resistant to standard biochemical techniques.

The neuromuscular junction (NMJ) is a 